\title{
Polyphenotypic Anaplastic Sarcoma of the Kidney: A Rare Case Report and Review of the Literature
}

\author{
Bita Geramizadeh, ${ }^{1,2,}{ }^{*}$ Ali Bahador, ${ }^{3}$ and Nadia Khalafi ${ }^{1}$ \\ ${ }^{1} \mathrm{MD}$, Department of Pathology \\ ${ }^{2}$ Transplant Research Center \\ ${ }^{3}$ MD Department of Pediatric Surgery \\ "Corresponding author: Bita Geramizadeh, Department of Pathology, Transplant Research Center, Shiraz University of Medical Sciences, Shiraz, IR Iran. Tel/Fax: +98-7136473238, \\ E-mail: geramib@gmail.com
}

Received 2017 April 02; Accepted 2017 May 14.

\begin{abstract}
Anaplastic sarcoma of the kidney is an extremely rare tumor, mostly observed in pediatric age group. This tumor is the counterpart of embryonal or undifferentiated sarcoma of the liver and pleuropulmonary blastoma of lung. To the best of the authors' knowledge, only 26 cases were reported in the English literature and the current case, a 3-year-old male, was the 27th reported case with this tumor. It is very important to differentiate this tumor from the Wilm tumor as well as renal cell carcinoma, as they were preoperative diagnosed in all of the reported cases.
\end{abstract}

\section{Introduction}

Common pediatric renal tumors are the Wilm tumor, clear cell sarcoma of the kidney, rhabdoid tumor, and mesoblastic nephroma (1). Anaplastic sarcoma of the kidney is an exceptionally rare tumor with polyphenotypic features; therefore, it is called polyphenotypic anaplastic sarcoma by some authors (2). Evaluation of the histologic and molecular characteristics of this tumor showed that it is very similar to undifferentiated (embryonal) sarcoma of the liver and pleuropulmonary blastoma of lung; therefore, some authors considered them as counterparts (3). To the best of the authors' knowledge, less than 26 cases with this tumor were reported in the English literature so far (4).

\section{Case Report}

A 3-year-old male presented with abdominal protrusion. He was the first child of healthy parents with no significant positive history. He was born through normal vaginal delivery and his first 3 years of life was uneventful.

His physical examination showed an abdominal mass; however, other parts including liver, spleen, heart, and lungs were normal. Laboratory findings were also unremarkable including complete blood count (CBC) as well as the liver and renal function tests.

Abdominal and pelvic ultrasonography showed normal liver and gallbladder. Left kidney was normal and right kidney showed a heterogeneous solid and cystic mass mostly in the upper part of kidney extending to middle part. The computed tomography(CT) scan of the abdomen showed a well-defined, solid cystic large mass with no calcification (Figure 1).

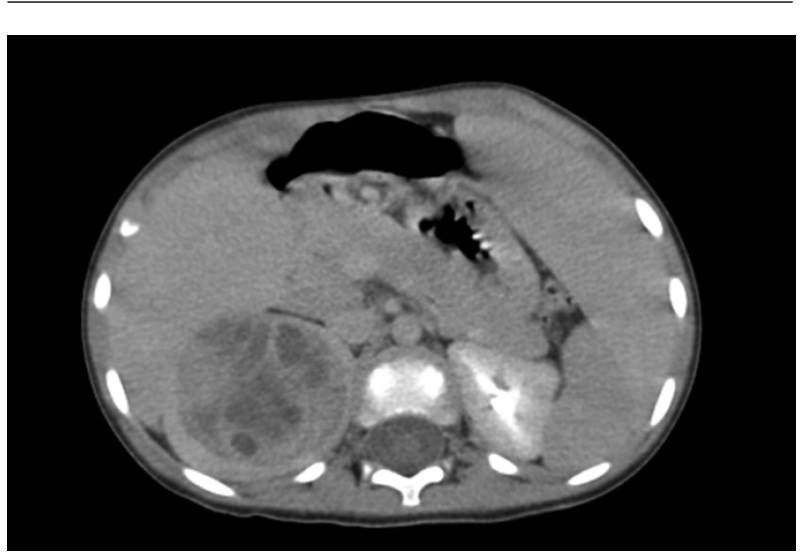

Figure 1. CT Scan Showed a Well-Defined Solid and Cystic Mass in the Right Kidney.

The patient was operated with the clinical diagnosis of the Wilm tumor, and radical nephrectomy with exploratory laparotomy was performed on him. The specimen in the pathology lab was a large kidney with bosselated surface. Cut section showed a round, creamy, and soft mass in the upper pole, measuring $5-\mathrm{cm}$ in the greatest diameter (Figure 2).

Microscopic sections of the mass showed a high grade tumor with highly atypical spindle cells. The tumor cells were hyperchromatic with indistinct cytoplasmic borders. There were also many intracytoplasmic PAS- (periodic acidSchiff) positive diastase resistant hyaline globules. Foci of rhabdomyoblastic differentiation as well as myxoid back- 


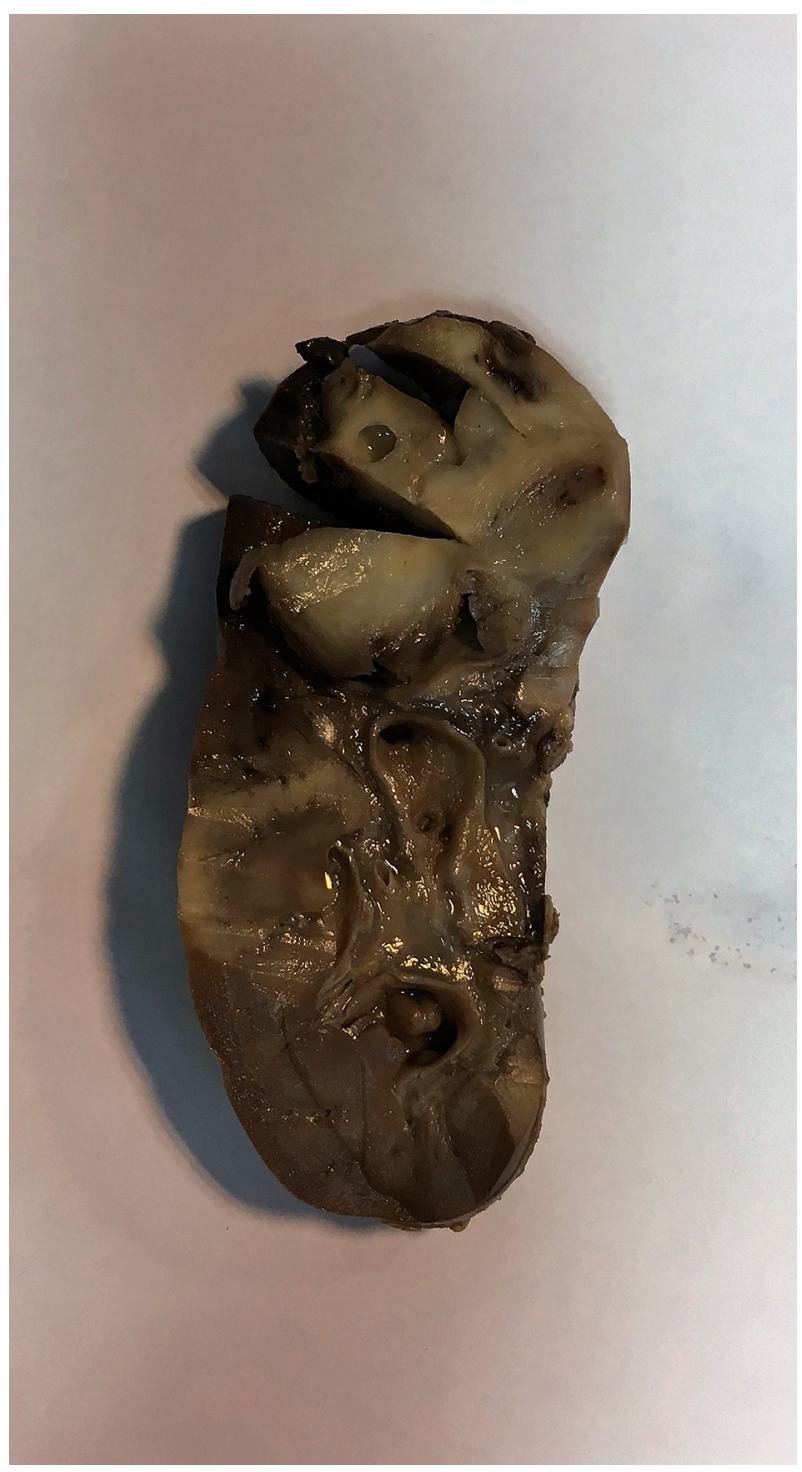

Figure 2. A Round Solid and Cystic Mass in the Upper Pole of the Kidney.

ground were observed. Tumor cells were reactive with vimentin, but nonreactive to all the other markers including cytokeratin, CD56, CD34, desmin, MIC2, MYOD1, SMA, and WT1. Mitotic numbers and proliferative index (Ki67) were very high in the above mentioned tumor cells. No epithelial marker was positive, and no chondroid and osteoid differentiation were identified (Figure 3A - D).

Following the diagnosis of anaplastic sarcoma of the kidney (similar to the Wilm tumor with unfavorable histology), chemotherapy was administered to the patient, and now after 3 months he is still well and under treatment.

\section{Discussion}

Renal tumors account for $6 \%$ to $8 \%$ of tumors in pediatric age group, the most common of which is the Wilm tumor; however, there are rare tumors, which are often missed with the incorrect common diagnosis of the Wilm tumor (2).

One of these rare pediatric tumors is anaplastic sarcoma of the kidney, which is an extremely rare tumor in this age group. To the best of the authors' knowledge, about 26 cases with this tumor were reported in the English literature so far (1-4). Table 1 shows the important characteristics of this tumor.

Table 1. Important Characteristics of the Reported Cases with Anaplastic Sarcoma of the Kidney

\begin{tabular}{ll}
\hline Finding & Characteristics \\
\hline Mean age, year & 12 (ranged 1 - 41) \\
\hline Female/male ratio & $1.6: 1$ \\
\hline Mean size, cm & 12 (ranged $4-22)$ \\
\hline Clinical presentation & Abdominal pain, mass, and protrusion \\
\hline Imaging & Solid, cystic, and heterogeneous \\
\hline Treatment & Surgery and chemotherapy \\
\hline Outcome & Poor, high risk of recurrence, and metastasis \\
\hline
\end{tabular}

The age range of the patients with this tumor is reported 1 to 41 years with the mean age of 12 years; however, in the 26 previously reported cases only 4 cases were above 18 , in the adult age group, and about half of them were below 5 . Most of the previously reported cases were female, and the female to male ratio was 1.6:1 $(1,4)$.

Majority of the patients presented with huge abdominal mass, abdominal pain, and protrusion; however, a few patients presented with hematuria. Mean size of the tumors in the reported cases was $12 \mathrm{~cm}$ that was quite a large size in a child (5).

According to the available literature, imaging studies was not specifically conducted on this tumor; however, in most of the cases the tumor was solid, cystic, and heterogeneous in ultrasonography, CT scan, and magnetic resonance imaging (MRI), which was completely compatible with the diagnosis of the Wilm tumor and could not be differentiated based on the imaging modalities (2).

Majority of the cases were operated with the incorrect diagnosis of the Wilm tumor or renal cell carcinoma. However, even pathologic misdiagnosis was common as the Wilm tumor or malignant mesenchymoma (1). Correct pathologic diagnosis can be made by considering specific features of the Wilm tumor including blastemal compo- 

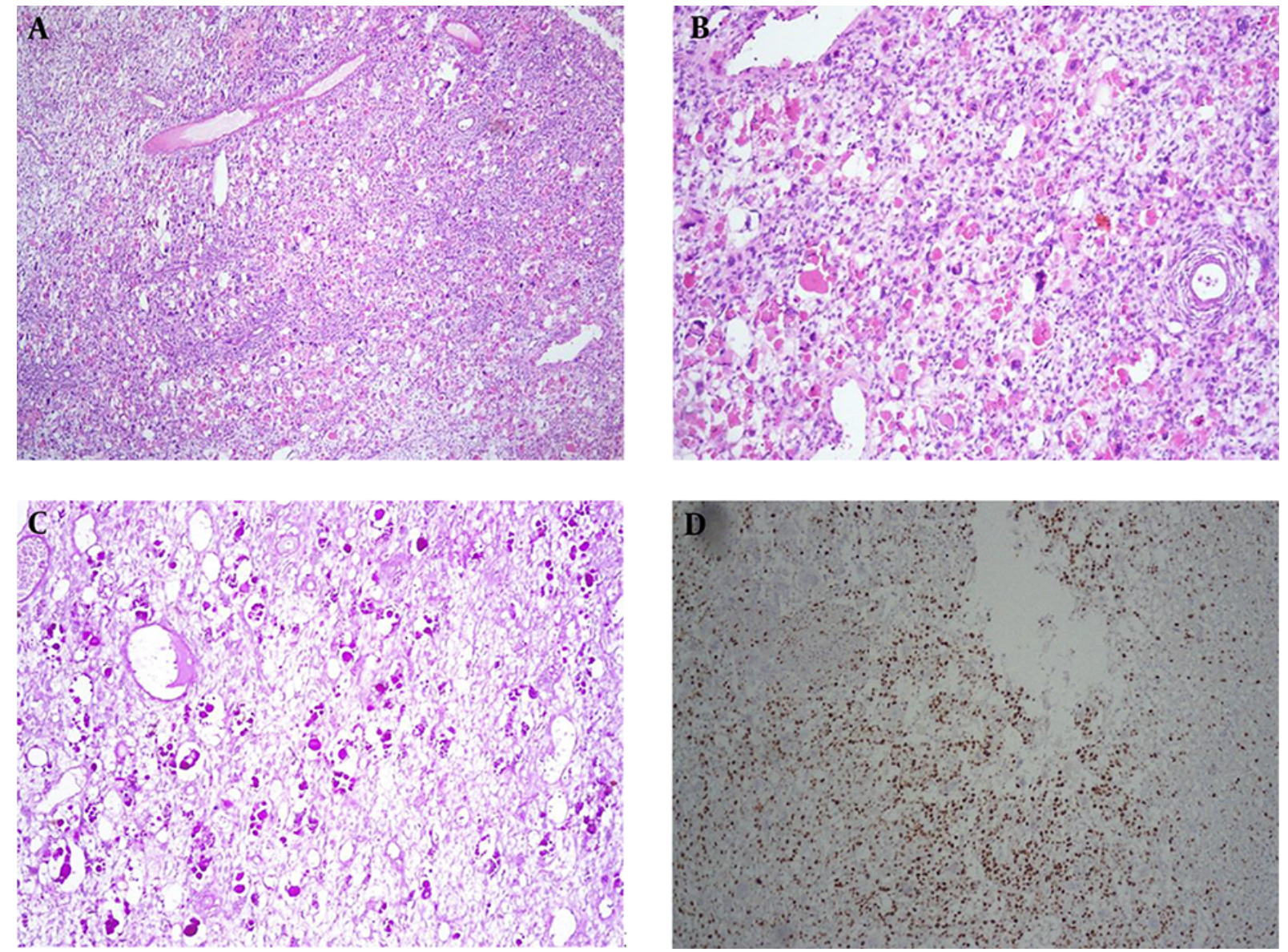

Figure 3. A, Sections From Liver Mass Showed Hypercellularity Composed of Hyperchromatic Nuclei with Severe Anisonucleosis and Many Mitotic (H\&E (H\&E staining; X100); B, Many Intra- and Extracellular Hyaline Globules Were Present; C, The Hyaline Globules Were PAS-Positive and Diastase Resistant; D, Ki-67 Was High.

nent and WT-1 positivity, which are not present in anaplastic sarcoma (2).

Another differential diagnosis can be the malignant mesenchymoma, which is composed of 2 components of sarcoma and mesenchymal differentiation to tissues such as cartilage. This tumor is known as a waste basket of soft tissue tumors in adult patients and many believe that this entity should be removed from the classifications of soft tissue tumors (1).

One of the most important points about this tumor is its relationship and histologic similarity to embryonal sarcoma of the liver previously emphasized; especially the presence of eosinophilic intracytoplasmic PAS-positive diastase resistant globules, which was completely unique and rarely mentioned in previous reports (5).

There is no definite recommendation about the chemotherapeutic regimen after nephrectomy; however, most of the cases were treated with the protocols of the Wilm tumor and some were treated very similar to primitive neuroectodermal tumor (PNET), and the Ewing sarcoma (6).

Overall, it seems that anaplastic sarcoma of the kidney has a poor prognosis with high risk of recurrence and lung or liver metastasis (7).

\section{References}

1. Vujanic GM, Kelsey A, Perlman EJ, Sandstedt B, Beckwith JB. Anaplastic sarcoma of the kidney: a clinicopathologic study of 20 cases of a new entity with polyphenotypic features. Am J Surg Pathol. 2007;31(10):145968. doi: 10.1097/PAS.0b013e31804d43a4. [PubMed: 17895746].

2. Labanaris A, Zugor V, Smiszek R, Nutzel R, Kuhn R. Anaplastic sarcoma of the kidney. ScientificWorldJournal. 2009;9:97-101. doi: 10.1100/tsw.2009.15. [PubMed:19219373].

3. Gomi K, Hamanoue S, Tanaka M, Matsumoto M, Kitagawa N, Niwa T, et al. Anaplastic sarcoma of the kidney with chromosomal abnormality: first report on cytogenetic findings. Hum Pathol. 2010;41(10):1495-9. doi: 10.1016/j.humpath.2010.03.008. [PubMed: 20656319]. 
4. Arabi H, Al-Maghraby H, Yamani A, Yousef Y, Huwait H. Anaplastic Sarcoma of the Kidney: A Rare Unique Renal Neoplasm. Int J Surg Pathol. 2016;24(6):556-61. doi:10.1177/1066896916639373. [PubMed: 27006300].

5. Wu MK, Goudie C, Druker H, Thorner P, Traubici J, Grant R, et al. Evolution of Renal Cysts to Anaplastic Sarcoma of Kidney in a Child With DICER1 Syndrome. Pediatr Blood Cancer. 2016;63(7):1272-5. doi: 10.1002/pbc.25959. [PubMed: 26928971].

6. Yoshida M, Hamanoue S, Seki M, Tanaka M, Yoshida K, Goto H, et al. Metachronous anaplastic sarcoma of the kidney and thyroid follicular carcinoma as manifestations of DICER1 abnormalities. Hum Pathol. 2017;61:205-9. doi: 10.1016/j.humpath.2016.06.024. [PubMed: 27697588].

7. Watanabe N, Omagari D, Yamada T, Nemoto N, Furuya T, Sugito K, et al Anaplastic sarcoma of the kidney: case report and literature review. Pediatr Int. 2013;55(5):e129-32. doi: 10.1111/ped.12167. [PubMed: 24134767]. 